\title{
Subspace Tracking Based Blind MIMO Transmit Preprocessing
}

\author{
W. Liu, L. L. Yang and L. Hanzo \\ School of ECS, University of Southampton, SO17 1BJ, United Kingdom. \\ Tel: +44-23-8059 6671, Fax: +44-23-8059 4508 \\ Email: \{wl03r,lly,lh\}@ecs.soton.ac.uk, http://www-mobile.ecs.soton.ac.uk
}

\begin{abstract}
In this contribution projection approximation subspace tracking using deflation (PASTD) is investigated in the context of MIMO transmit preprocessing systems by exploiting the specific property of Time Division Duplexing (TDD) techniques that the uplink and downlink channels are similar, since they both use the same carrier frequency. Hence the channel estimated from the received signal can also be used for transmit preprocessing. More explicitly, based on the received signal, the PASTD algorithm is used for tracking both the left and the right singular vectors of the MIMO channel matrix, which are required by eigenmode transmissions, instead of periodically reestimating the MIMO channel matrix and performing the singular value decomposition (SVD), which would impose a high computational complexity. A specific deficiency of the family of subspace tracking algorithms is their phase ambiguity imposed by the the non-unique nature of the SVD, which is resolved in this treatise by employing differential encoding. The efficiency of the proposed subspace tracking scheme is demonstrated by our performance results, indicating that the advocated technique preforms within 1dB from the BER curve of the perfect channel estimation aided benchmarker.
\end{abstract}

\section{INTRODUCTION}

Due to the emerging high demand for supporting novel multimedia applications, next generation wireless systems are expected to support higher data rates. When employing multiple antennas at both the transmitter and receiver, multiple input multiple output (MIMO) systems have the potential achieving a high transmission rate than their traditional single input single output (SISO) counterparts [1].

MIMO systems have attracted intensive research interests during the last decade [1]-[3]. In the absence of channel state information (CSI) at the transmitter, space time coding [4] or spatial multiplexing [5], [6] constitue prime candidates for MIMO transmission. However, when the CSI is available at both the transmitter and the receiver, a more sophisticated technique refered to as eigenmode transmission [7] can be used for decomposing the MIMO channel into several independent SISO subchannels, which involves the singular value decomposition (SVD) of the MIMO channel matrix. In this case no joint detection is needed and the resultant single-antenna-based detection algorithm becomes rather simple.

The third-generation (3G) wireless systems support two different modes, namely frequency division duplexing (FDD) and time division duplexing (TDD) [8], [9]. In the FDD mode, the uplink (UL) and downlink (DL) signals are transmitted at

The financial support of the European Union under the auspices of the Newcom and Phoenix projects, as well as that of the EPSRC UK is gratefully acknowledged. different carrier frequencies, which results in independently fading channels for the UL and DL. By contrast, in the TDD mode, the UL and DL transmissions ensue at the same carrier frequency. Hence the UL and DL channels tend to fade together and therefore can be considered as similar [8]. We will exploit this similarity of the UL and DL channels of the TDD mode in this paper.

The CSI required at the transmitter can be obtained with the aid of the side-information control channel from the receiver in the FDD mode. Alternatively, it can be directly estimated on the basis of the received signal's quality and exploited by the transmitter in the TDD mode.

Channel estimation (CE) followed by singular value decomposion (SVD) is invoked, when eigenmode transmissions are employed [10], which potentially imposes a high computational complexiy. Instead of estimating the entire MIMO channel matrix and then additionally implementing SVD, it was claimed in [11], [12] that subspace tracking based algorithms may result in lower computational complexity in the context of eigenmode transmissions.

In the family of subspace tracking algorithms, the so-called projection approximation tracking combined with deflation (PASTD) [13] has been shown to be applicable in diverse scenarios [14], [15]. Hence, in this paper PASTD algorithm is employed for subspace tracking in a MIMO-aided TDD system.

\section{MIMO TRANSMISSION MODEL}

Consider a system having $M_{T}$ transmitter and $M_{R}$ receiver antennas subjected to a flat-fading channel between any pair of transmitter and receiver antennas. Then the received $M_{R^{-}}$ dimensional symbol vector $\mathbf{y}$ can be expressed as

$$
\mathbf{y}=\mathbf{H} \mathbf{x}+\mathbf{n},
$$

where $\mathbf{x}$ is the $M_{T}$-dimensional transmitted symbol vector and $\mathbf{H}$ is an $\left(M_{R} \times M_{T}\right)$-dimensional complex channel matrix with the $(i, j)$ th element being the fading channel between the $i$ th receive and $j$ th transmit antennas. Finally, $\mathbf{n}$ is the $M_{R}$-dimensional AWGN vector having a zero-mean and $E\left(\mathbf{n n}^{H}\right)=\sigma_{n}^{2} \mathbf{I}_{M_{R}}$. Here $\mathbf{I}_{M}$ is an $(M \times M)$-dimensional identity matrix.

If the rank of $\mathbf{H}$ is assumed to be $q(q \leq \min (M, N))$, the SVD of the channel matrix $\mathbf{H}$ is given by

$$
\begin{aligned}
\mathbf{H} & =\mathbf{U} \boldsymbol{\Lambda} \mathbf{V}^{H} \\
& =\left[\mathbf{U}_{s} \mathbf{U}_{n}\right]\left[\begin{array}{cc}
\boldsymbol{\Lambda}_{s} & \mathbf{0} \\
\mathbf{0} & \mathbf{0}
\end{array}\right]\left[\begin{array}{c}
\mathbf{V}_{s}^{H} \\
\mathbf{V}_{n}^{H}
\end{array}\right],
\end{aligned}
$$


where $\mathbf{U}$ is an $\left(M_{R} \times M_{R}\right)$-dimensional unitary matrix satisfying $\mathbf{U}^{H} \mathbf{U}=\mathbf{I}_{M_{R}}$ and $\mathbf{V}$ is an $\left(M_{T} \times M_{T}\right)$-dimensional unitary matrix having the property of $\mathbf{V}^{H} \mathbf{V}=\mathbf{I}_{M_{T}}$, while $\boldsymbol{\Lambda}$ is an $\left(M_{R} \times M_{T}\right)$-dimensional matrix and $\mathbf{I}_{M}$ is an $(M \times M)$ dimensional identity matrix. In the second line of (2), $\boldsymbol{\Lambda}_{s}$ is a $(q \times q)$-dimensional diagonal matrix having diagonal elements of $\lambda_{1} \geq \lambda_{2} \cdots \lambda_{q-1} \geq \lambda_{q}$, which are the singular values of $\mathbf{H}$. Furthermore, in (2) we portrayed $\mathbf{U}$ and $\mathbf{V}$ in form of two components, where $\mathbf{U}_{s}$ is an $\left(M_{R} \times q\right)$ dimensional matrix constituted by the first $p$ columns of $\mathbf{U}$, which span the column-space of $\mathbf{H}$ and $\mathbf{V}_{s}$ is an $\left(M_{T} \times p\right)$ dimensional matrix formed by the first $q$ columns of $\mathbf{V}$, which span the row-space of $\mathbf{H}$. Still referring to (2), $\mathbf{U}_{n}$ is an $\left[M_{R} \times\left(M_{R}-q\right)\right]$-dimensional matrix, which is orthogonal to $\mathbf{U}_{s}$, while spanning the null space of $\mathbf{H}$ and $\mathbf{V}_{n}$ is an $\left[M_{T} \times\left(M_{T}-q\right)\right]$-dimensional matrix that is orthogonal to $\mathbf{V}_{s}$ and spans the left null space of $\mathbf{H}$.

If the channel matrix $\mathbf{H}$ is known at both the transmitter and receiver, the so-called eigenmode transmission regime of [7] can be invoked to decompose the MIMO channel into orthogonal subchannels by applying $\mathbf{V}_{s}$ and $\mathbf{U}_{s}$ at the transmitter and receiver, respectively, yielding

$$
\begin{aligned}
\tilde{\mathbf{y}} & =\mathbf{U}_{s}^{H} \mathbf{y} \\
& =\mathbf{U}_{s}^{H}\left(\mathbf{H} \mathbf{V}_{s} \tilde{\mathbf{x}}+\mathbf{n}\right) \\
& =\boldsymbol{\Lambda} \tilde{\mathbf{x}}+\tilde{\mathbf{n}}
\end{aligned}
$$

where $\tilde{\mathbf{x}}$ is a $q$-dimensional transmitted symbol vector, while $\tilde{\mathbf{n}}=\mathbf{U}_{s}^{H} \mathbf{n}$ is a $q$-dimensional noise vector, which has the same statistical properties as $\mathbf{n}_{(d l, k)}$, because $\mathbf{U}_{s}^{H}$ is a unitary matrix.

As an explicit benefit of using the SVD, the known channel matrix $\mathbf{H}$ is finally decomposed into $q$ independent orthogonal subchannels, each of which has a channel gain of $\lambda_{i}$ and this transmit preprocessing regime is referred to as eigenmode transmission [7].

As a further simplication, it was shown in [11] that highintegrity reception can be achieved, if we opt for transmitting in a limited number of $p(1 \leq p \leq q)$ subchannels having channel gains of $\lambda_{1} \geq \lambda_{2} \cdots \geq \lambda_{p}$ for achieving a high throughput, while meeting the specific target BER performance.

Another potential advantage of eigenmode transmission is that only the left singular vectors of $\mathbf{U}_{s}$ and the right singular vectors of $\mathbf{V}_{s}$ are needed, as we can see in (3). Hence it is intuitively appealing to invoke algorithms, which estimate or update the singular vectors only [11], [12] instead of estimating the entire MIMO channel matrix $\mathbf{H}$ and then additionally implementing the SVD, which would inevitably impose a high computational complexity.

\section{TDD MIMO TRANSMISSION MODEL}

A full-duplex MIMO link may be created using either FDD or TDD mode. In this paper, we assume employing the TDD mode. Consider a TDD system using $M_{T}$ antennas at the base station (BS) and $M_{R}$ antennas at the mobile station (MS), encountering a flat-fading channel between any pair of transmitter and receiver antennas. Furthermore, for simplicity, we assume that the system supports a single user. Then the $M_{R}$-dimensional received symbol vector $\mathbf{y}_{d l}(k)$ of the $\mathrm{DL}$ and the $M_{T}$-dimensional received symbol vector $\mathbf{y}_{u l}(k)$ of the UL can be expressed as

$$
\begin{aligned}
& \mathbf{y}_{d l}(k)=\mathbf{H}_{d l}(k) \mathbf{x}_{d l}(k)+\mathbf{n}_{d l}(k), \\
& \mathbf{y}_{u l}(k)=\mathbf{H}_{u l}(k) \mathbf{x}_{u l}(k)+\mathbf{n}_{u l}(k),
\end{aligned}
$$

where $\mathbf{x}_{d l}(k)$ is an $M_{T}$-dimensional DL symbol vector transmitted from the BS to the MS, while $\mathbf{x}_{u l}(k)$ is an $M_{R^{-}}$ dimensional UL symbol vector transmitted from the MS to the BS. Furthermore, $\mathbf{H}_{d l}(k)$ is the DL channel matrix and $\mathbf{H}_{u l}(k)$ is the UL channel matrix. Moreover, $\mathbf{n}_{d l}(k)$ is the DL AWGN noise vector having a zero-mean and $E\left(\mathbf{n}_{d l} \mathbf{n}_{d l}^{H}\right)=\sigma_{\mathbf{n}_{d l}}^{2} \mathbf{I}_{M_{R}}$, $\mathbf{n}_{u l}(k)$ is the UL AWGN noise vector having a zero-mean and $E\left(\mathbf{n}_{u l} \mathbf{n}_{u l}^{H}\right)=\sigma_{\mathbf{n}_{u l}}^{2} \mathbf{I}_{M_{T}}$.

Since the UL and DL timeslots of a TDD link are transmitted on the same carrier frequency, the UL and DL channel matrices may be assumed to be identical, provided that the Doppler frequency is sufficiently low and hence the corresponding channel impulse response (CIR) does not change dramatically during the time between the UL and DL time slot. Hence we have

$$
\mathbf{H}_{u l}(k)=\mathbf{H}_{d l}^{T}(k) .
$$

Upon substituting (6) into (5), we arrive at

$$
\mathbf{y}_{u l}(k)=\mathbf{H}_{d l}^{T}(k) \mathbf{x}_{u l}(k)+\mathbf{n}_{u l}(k) .
$$

The transmitted symbol vector $\mathbf{x}_{u l}$ is conjugated before transmission, as proposed in [11]. In this case, we obtain

$$
\mathbf{y}_{u l}(k)=\mathbf{H}_{d l}^{T}(k) \mathbf{x}_{u l}^{*}(k)+\mathbf{n}_{u l}(k) .
$$

Furthermore, the received symbol vector is conjugated as well, hence we have

$$
\mathbf{y}_{u l}^{*}(k)=\mathbf{H}_{d l}^{H}(k) \mathbf{x}_{u l}(k)+\mathbf{n}_{u l}^{*}(k) .
$$

According to (2), the SVD of $\mathbf{H}_{d l}$ can be expressed as

$$
\mathbf{H}_{d l}=\left[\begin{array}{ll}
\mathbf{U}_{d l_{s}} & \mathbf{U}_{d l_{n}}
\end{array}\right]\left[\begin{array}{cc}
\boldsymbol{\Lambda}_{d l_{s}} & \mathbf{0} \\
\mathbf{0} & \mathbf{0}
\end{array}\right]\left[\begin{array}{c}
\mathbf{V}_{d l_{s}}^{H} \\
\mathbf{V}_{d l_{n}}^{H}
\end{array}\right]
$$

where $\mathbf{U}_{d l_{s}}$ is an $\left(M_{R} \times q\right)$-dimensional unitary matrix, while $\mathbf{V}_{d l_{s}}$ is an $\left(M_{T} \times q\right)$-dimensional unitary matrix. Furthermore, $\boldsymbol{\Lambda}_{d l_{s}}$ is a $(q \times q)$-dimensional diagonal matrix with its diagonal elements given by $\lambda_{1} \geq \lambda_{2} \cdots \lambda_{q-1} \geq \lambda_{q}$, which are the singular values of $\mathbf{H}_{d l}$. Accordingly, the SVD of $\mathbf{H}^{H}$ is given by

$$
\mathbf{H}_{d l}^{H}=\left[\begin{array}{ll}
\mathbf{V}_{d l_{s}} & \mathbf{V}_{d l_{n}}
\end{array}\right]\left[\begin{array}{cc}
\boldsymbol{\Lambda}_{d l_{s}} & 0 \\
0 & 0
\end{array}\right]\left[\begin{array}{c}
\mathbf{U}_{d l_{s}}^{H} \\
\mathbf{U}_{d l_{n}}^{H}
\end{array}\right] .
$$

When eigenmode transmission is used for the sake of avoiding interference among the transmitted data symbols, the $p$-dimensional transmitted symbol vectors $\tilde{\mathbf{x}}_{d l}$ and $\tilde{\mathbf{x}}_{u l}$ are multiplied by $\mathbf{V}_{d l_{s p}}$ and $\mathbf{U}_{d l_{s p}}$ given by the first $p$ columns of $\mathbf{V}_{d l_{s}}$ and $\mathbf{U}_{d l_{s}}$, respectively, before their transmission. Accoding to (3), we obtain

$$
\mathbf{y}_{d l}(k)=\mathbf{H}_{d l}(k) \mathbf{V}_{d l_{s p}} \tilde{\mathbf{x}}_{d l}+\mathbf{n}_{d l}(k),
$$




$$
\mathbf{y}_{u l}^{*}(k)=\mathbf{H}_{d l}^{H}(k) \mathbf{U}_{d l_{s p}} \tilde{\mathbf{x}}_{u l}+\mathbf{n}_{u l}(k) .
$$

The resultant received symbol vectors $\mathbf{y}_{d l}$ and $\mathbf{y}_{u l}^{*}$ are multiplied by the matrices $\mathbf{U}_{d l_{s p}}^{H}$ and $\mathbf{V}_{d l_{s p}}^{H}$, respectively, for the sake of avoiding interference among the transmitted data symbols. Finally, we obtain

$$
\begin{aligned}
\tilde{\mathbf{y}}_{d l}(k) & =\mathbf{U}_{d l_{s p}}^{H}(k) \mathbf{y}_{d l}(k) \\
& =\boldsymbol{\Lambda}_{d l_{p}}(k) \tilde{\mathbf{x}}_{d l}(k)+\mathbf{U}_{d l_{s p}}^{H}(k) \mathbf{n}_{d l}(k), \\
\tilde{\mathbf{y}}_{u l}(k) & =\mathbf{V}_{d l_{s p}}^{H}(k) \mathbf{y}_{u l}^{*}(k) \\
& =\boldsymbol{\Lambda}_{d l_{p}}(k) \tilde{\mathbf{x}}_{u l}(k)+\mathbf{V}_{d l_{s p}}^{H}(k) \mathbf{n}_{u l}(k),
\end{aligned}
$$

where $\boldsymbol{\Lambda}_{d l_{p}}$ is a $(p \times p)$-dimensional diagonal matrix having $\lambda_{1} \geq \lambda_{2} \cdots \lambda_{p-1} \geq \lambda_{p}$ as its diagonal elements. As we can see, only the matrix $\mathbf{U}_{d l_{s p}}$ has to be known at the MS, while the matrix $\mathbf{V}_{d l_{s p}}$ is used for preprocessing at the BS.

The matrices $\mathbf{U}_{d l_{s p}}$ and $\mathbf{V}_{d l_{s p}}$ can be obtained by SVD of the channel matrix $\mathbf{H}_{d l}$. However, this requires estimating the channel matrix first, then implementing the SVD, which improses a high computational complexity. Observe in (9) to (15) however, that only the subspace matrices $\mathbf{U}_{d l_{s p}}$ and $\mathbf{V}_{d l_{s p}}$ are required instead of the knowledge of the entire channel matrix.

Let us continue by considering the DL transmission in more detail. More explicitly, our goal is to obtain the matrices $\mathbf{U}_{d l_{s p}}$ and $\mathbf{V}_{d l_{s p}}$ without estimating the channel matrix $\mathbf{H}$ and without performing the SVD of $\mathbf{H}$. Upon substituting (10) into (12), we obtain

$$
\mathbf{y}_{d l}=\left[\begin{array}{ll}
\mathbf{U}_{d l_{s}} & \mathbf{U}_{d l_{n}}
\end{array}\right]\left[\begin{array}{cc}
\boldsymbol{\Lambda}_{d l_{s}} & \mathbf{0} \\
\mathbf{0} & \mathbf{0}
\end{array}\right]\left[\begin{array}{c}
\mathbf{V}_{d l_{s}}^{H} \\
\mathbf{V}_{d l_{n}}^{H}
\end{array}\right] \mathbf{V}_{d l_{s p}} \tilde{\mathbf{x}}_{d l}+\mathbf{n}_{d l}
$$

The autocorrelation matrix of the vector $\mathbf{y}_{d l}$ of received symbols is given by

$$
\mathbf{R}_{\mathbf{y}_{d l}}=E\left[\mathbf{y}_{d l} \mathbf{y}_{d l}^{H}\right]=\mathbf{H}_{d l} \mathbf{V}_{d l_{s p}} \mathbf{R}_{\tilde{\mathbf{x}}_{d l}} \mathbf{V}_{d l_{s p}}^{H} \mathbf{H}_{d l}^{H}+\sigma_{\mathbf{n}_{d l}}^{2} \mathbf{I} .
$$

Let the total average transmit power $P$ be a constant and let us allocate an equal power to each nonzero subchannel in (16). Then we obtain the autocorrelation of the $p$-dimensional vector $\tilde{\mathbf{x}}_{d l}$ of transmitted symbols as follows

$$
\mathbf{R}_{\tilde{\mathbf{x}}_{d l}}=E\left[\tilde{\mathbf{x}}_{d l} \tilde{\mathbf{x}}_{d l}^{H}\right]=\frac{P}{p} \mathbf{I}_{p} .
$$

Hence, following a few further manipulations, (17) can be written as

$\mathbf{R}_{\mathbf{y}_{d l}}=\left[\begin{array}{ll}\mathbf{U}_{d l_{s}} & \mathbf{U}_{d l_{n}}\end{array}\right]\left[\begin{array}{cc}\frac{P}{p} \boldsymbol{\Lambda}_{d l_{s p}}^{2}+\sigma_{\mathbf{n}_{d l}}^{2} & \mathbf{0} \\ \mathbf{0} & \sigma_{\mathbf{n}_{d l}}^{2}\end{array}\right]\left[\begin{array}{c}\mathbf{U}_{d l_{s}}^{H} \\ \mathbf{U}_{d l_{n}}^{H}\end{array}\right]$

where $\mathbf{U}_{d l_{s}}$ is constituted by $q$ eigenvectors of $\mathbf{R}_{\mathbf{y}_{d l}}$ associated with the $q$ largest eigenvalues $\left(\frac{P}{p} \lambda_{1}^{2}+\sigma_{\mathbf{n}_{d l}}^{2}\right) \geq\left(\frac{P}{p} \lambda_{2}^{2}+\right.$ $\left.\sigma_{\mathbf{n}_{d l}}^{2}\right) \cdots \geq\left(\frac{P}{p} \lambda_{q}^{2}+\sigma_{\mathbf{n}_{d l}}^{2}\right)$ of $\mathbf{R}_{\mathbf{y}_{d l}}$. The space spanned by the columns of $\mathbf{U}_{d l_{s}}$ is referred to as the signal subspace, while $\mathbf{U}_{d l_{n}}$ consists of $\left(M_{R}-q\right)$ number of eigenvectors of $\mathbf{R}_{\mathbf{y}_{d l}}$ related to $\left(M_{R}-q\right)$ number of eigenvalues $\left\{\sigma_{\mathbf{n}_{d l}}^{2}\right\}$ of $\mathbf{R}_{\mathbf{y}_{d l}}$. Finally, the space spanned by the columns of $\mathbf{U}_{d l_{n}}$ is

\begin{tabular}{|l|}
\hline Operation procedure of PASTD algorithm \\
\hline $\mathbf{y}_{1}(k)=\mathbf{y}_{d l}(k)$ \\
For $i=1,2, \cdots, p$, Do \\
$r_{i}(k)=\mathbf{w}_{i}(k-1) \mathbf{y}_{i}(k)$; prejection operation \\
$d_{i}(k)=\beta d_{i}(k-1)+\left|r_{i}\right|^{2} ;$ \\
$\mathbf{e}_{i}(k)=\mathbf{y}_{i}(k)-\mathbf{w}_{i}(k-1) r_{i}(k) ;$ \\
$\mathbf{w}_{i}(k)=\mathbf{w}_{i}(k-1)+\mathbf{e}_{i}(k)\left[r_{i}^{*}(k) / d_{i}(t)\right] ;$ updating eigenvectors \\
$\mathbf{y}_{i+1}(k)=\mathbf{y}_{i}(k)-\mathbf{w}_{i}(k) r_{i}(k) ;$ deflation
\end{tabular}

TABLE I

THE PASTD ALGORITHM DESIGNED FOR TRACKING THE SIGNAL SUBSPACE COMPONENTS OF THE RECEIVED SIGNAL VECTOR $\mathbf{y}_{d l}$

termed as the noise subspace, which is orthogonal to the signal subspace [12], [14].

We can see from our discussions above that the eigenvectors in $\mathbf{U}_{d l_{s}}$ also consist of the orthonormal basis vector of the column-space of $\mathbf{H}_{d l}$. Moreover, when the vector $\mathbf{y}_{d l}$ of received symbols becomes available, so-called subspace tracking algorithms can be used to track the orthonormal basis vectors of $\mathbf{U}_{d l_{s p}}$, which spans the column-space of $\mathbf{H}$.

Similarly, when the vector $\mathbf{y}_{u l}^{*}$ of received symbols becomes available, the eigenvectors in $\mathbf{V}_{d l_{s p}}$ can be tracked as well, which spans the row-space of $\mathbf{H}$. Upon obtaining the corresponding left and right singular vectors of $\mathbf{H}$, the eigenmode MIMO-aided transmission regime described above can be employed.

In the family of different subspace tracking algorithms, the Projection Approximation Subspace Tracking technique using deflation (PASTD) [13] stands out as one of the most popular algorithms. In the next section, the PASTD algorithm will be briefly described in the context of tracking the elements of $\mathbf{U}_{d l_{s p}}$. The same algorithm can also be used for tracking the elements of $\mathbf{V}_{d l_{s p}}$.

\section{PASTD SubsPaCe TRACKING [13]}

The PASTD algorithm of [13] designed for signal subspace tracking is summarized in Table I, where $\mathbf{y}_{d l}(k)$ is the $k$ th $M_{R}$-dimensional received signal vector generated for DL transmission, while $d_{i}(k)$ represents the exponentially weighted estimate of the $i$ th eigenvalue and $\mathbf{w}_{i}(k)$ denotes the estimate of the $i$ th eigenvector at the $k$ th time instant. Furthermore, $\beta(0<\beta \leq 1)$ represents the forgetting factor. Table I summarizes the operations of the PASTD algorithm, which is based on the so-called deflation technique [13] and its basic philosophy is that of the sequential estimation of the so-called principal components [13]. The most dominant eigenvector is updated first by applying the PAST algorithm at the 1st iteration [13]. Then the projection of the current signal sample vector $\mathbf{y}_{d l}(k)$ onto this eigenvector is removed from $\mathbf{y}_{d l}(k)$ itself. Now the second most dominant eigenvector becomes the most dominant one in the updated signal vector and hence can be extracted in the same way as outlined above. This procedure is applied repeatedly, until all desired eigencomponents have been estimated.

Since the deflation [13] technique results in a strong loss of orthonormality between the singular vectors [13], the GramSchmidt orthonormalization [16] technique is invoked for 


\begin{tabular}{|l|r|}
\hline Number of transmitter antennas $M_{T}$ & 4 \\
\hline Number of receiver antennas $M_{R}$ & 4 \\
\hline Normalized maximum Doppler frequency $f_{d m} T_{s}$ & 0.001 \\
\hline Forgetting factor $\beta$ in Section IV & 0.95 \\
\hline
\end{tabular}

TABLE II

PARAMETERS FOR THE PASTD ALGORITHM IN TDD MODE

reorthogonalizing the signal subspace after each update.

The variables $d_{i}(0)$ and $\mathbf{w}_{i}(0)$ have to be initialized, as seen in Table I . Specifically, the SVD of the first $M$ vectors of the received symbols are used for the initialization of $d_{i}(0)$ and $\mathbf{w}_{i}(0)$ [14].

Since the singular vector generated according to (2) can be different up to a complex-valued coefficient of unit norm [11], it may cause phase ambiguity [11], which can be resolved for example by differential encoding, leading to differential phase shift keying (DPSK) modulation [11].

\section{Performance Results}

Having described the TDD system and the PASTD algorithms in Section IV, in this section our simulation results are provided in order to characterize the attainable performance of PASTD subspace tracking in the context of a TDD system. Furthermore, differential BPSK modulation is used.

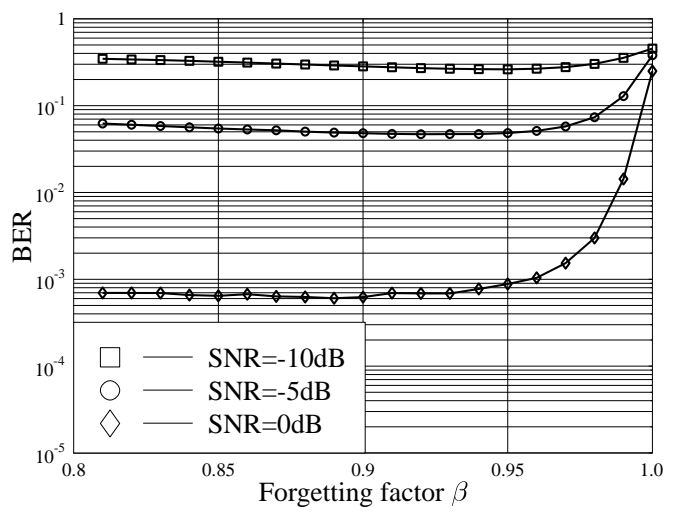

Fig. 1. BER performance against the forgetting factor $\beta$ introduced in Section IV, when only the largest eigenvalue is used for uplink transmission at different values of SNR. The remaining parameters are the same as in Table II.

In Figure 1 the achievable BER performance is plotted against the forgetting factor $\beta$, when only the largest eigenvalue is used for both the uplink and downlink transmissions, respectively, at SNRs of $-10 \mathrm{~dB},-5 \mathrm{~dB}$ and $0 \mathrm{~dB}$. The remaining parameters are the same as in Table II. We can see from Figure 1 that for a given SNR, the BER slightly decreases upon increasing the forgetting factor $\beta$, until an optimum point is reached. Beyond this point the BER increases relatively sharply upon increasing the forgetting factor $\beta$. This is because for the low normalized Doppler frequency of $f_{d m} T_{s}=0.001$, the channel exhibits a high correlation for a long period, which allows us to exploit the channel knowledge over a longer period, resulting in a higher forgetting factor. Beyond the optimum value of $\beta$, more than necessary past channel output samples are invoked, therefore the correlation between a far distant channel sample and the current one is low and hence the effects of the noise imposed by a distant noisy sample on the correlation becomes more dominant, which actually degrades the algorithm's performance. We can also see for $\mathrm{SNR}=-10 \mathrm{~dB}$ and $-5 \mathrm{~dB}$ that the optimum forgetting factor is around $\beta=0.95$, while for $\mathrm{SNR}=0 \mathrm{~dB}$ it is around 0.90 . The reason behind this may be attributed to the observation that for lower SNRs a higher number of noisy samples may be needed to mitigate the effects of the noise and hence a higher forgetting factor is required. By contrast, for higher SNRs a lower number of noisy samples is sufficient for mitigating the effects of noise, which results in a lower forgetting factor $\beta$.

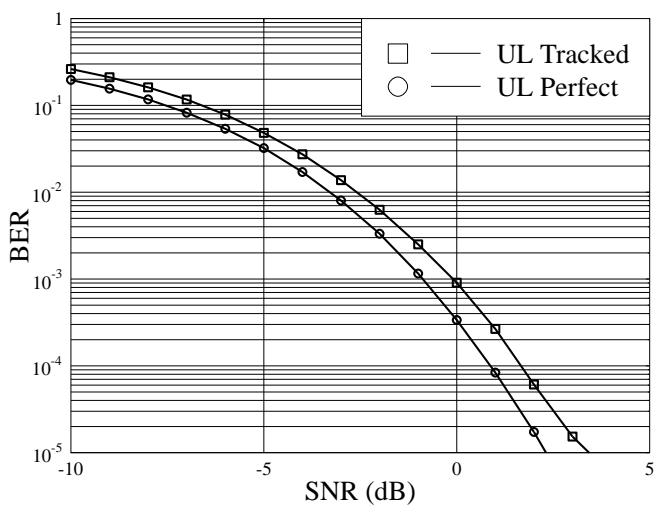

Fig. 2. BER versus SNR performance, when only the largest eigenvalue is used for uplink transmission. The remaining parameters are the same as in Table II.

In Figure 2 the attainable BER performance is portrayed for different values of the SNR, when only the largest eigenvalue is used for uplink transmission. The remaining parameters are the same as in Table II. We can see from Figure 2 that the achievable BER performance of PASTD subspace tracking is similar to that achieved with the aid of perfect channel knowledge. Observe, however that the BER difference between the perfect estimation based scenario and the tracked scenario becomes higher upon increasing the SNR. This is because the forgetting factor of $\beta=0.95$ is not the optimum value for higher SNRs, as seen earlier in Figure 1. The same performance is observed for downlink transmissions because the uplink and downlink are similar.

In Figure 3 the achievable mean BER performance is plotted against the forgetting factor $\beta$ introduced in Section IV, when the first two largest eigenvalues are used for UL transmissions for different values of the SNR. The remaining parameters are the same as in Table II. We can see from Figure 3 that different optimum forgetting factors are found for the different values of the SNR. The reason for this observation is the same as that stated earlier for Figure 1. 


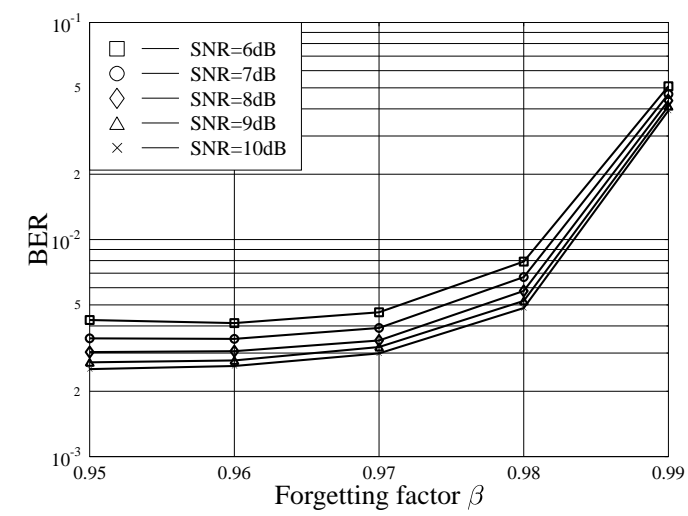

Fig. 3. Mean BER performance against the forgetting factor $\beta$ in Section IV, when the first two largest eigenvalues are used for uplink transmission at different SNRs. The remaining parameters are the same as in Table II.

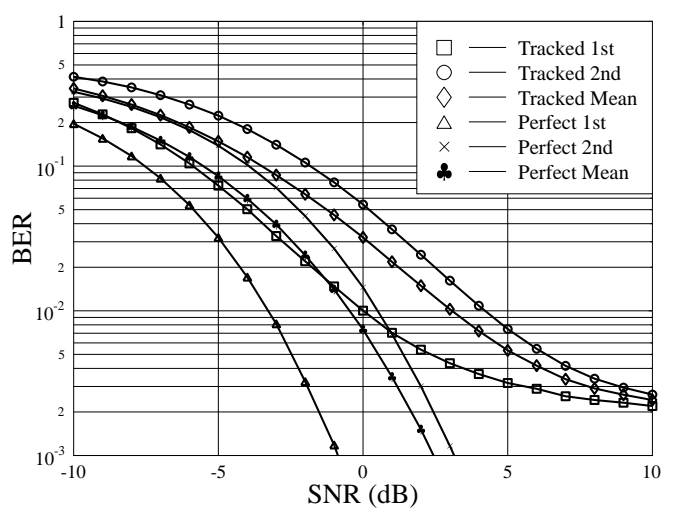

Fig. 4. BER versus SNR performance, when the first two eigenvalues are used for uplink transmission. The remaining parameters are the same as in Table II.

In Figure 4 the attainable BER performance is characterized for different values of the SNR, when the first two eigenvalues are used for uplink transmission. The remaining parameters are the same as in Table II. We can see from Figure 4 that the performance recorded for the largest eigenvalue is the same as in Figure 4 and when the availability of perfect channel knowledge is assumed, while the performances achieved by the PATSD algorithm are quite different from theoe seen in Figure 2. This is because the subspace tracking algorithm is unable to eliminate the interferece between the two eigenvalues, while there is no interference between the two eigenvalues in case of perfect channel knowledge. Furthermore, the BER curve exhibits a floor value upon increasing the SNR. This is because the interference between two eigenvalues becomes the dominant factor for high values of the SNR.

\section{SUMMARY AND CONCLUSIONS}

In this paper, PASTD subspace tracking aided MIMO transmit processing techniques were investigated in the context of a TDD system. Since only the left or right singular vectors of the channel matrix are required at transmitter and receiver, respectively, for eigenmode transmission in the TDD mode, PASTD subspace tracking can be used at both the transmitter and receiver to acquire the required left and right singular vectors without estimating the entire MIMO channel matrix $\mathbf{H}$. This operation is followed by SVD of $\mathbf{H}$, which typically results in a high complexity. Furthermore, since the PASTD subspace tracking technique is a blind algorithm, it improves the achievable spectral efficiency. A specific deficiency of the family of subspace tracking algorithms is their phase ambiguity imposed by the the non-unique nature of SVD, which was resolved by employing differential encoding. Finally, the efficiency of the proposed scheme was verified by our simulations.

\section{REFERENCES}

[1] A. J. Paulraj, D. A. Gore, R. U. Nabar, and H. Bölcskei, "An overview of MIMO communications - a key to gigabit wireless," Proceedings of the IEEE, vol. 92, pp. 198- 218, February 2004.

[2] D. Gesbert, M. Shafi, D. Shiu, P. J. Smith, and A. Naguib, "From theory to practice: an overview of MIMO space-time coded wireless systems," IEEE Journal on Selected Areas in Communications, vol. 21, pp. 281302, April 2003.

[3] S. N. Diggavi, N. Al-dhahir, A. Stamoulis, and A. R. Calderbank, "Great expectations: the value of spatial diversity in wireless networks," Proceedings of the IEEE, vol. 92, pp. 219- 270, February 2004.

[4] S. M. Alamouti, "A simple transmit diversity technique for wireless communications," IEEE Journal on Selected Areas in Communications, vol. 16, pp. 1451-1458, October 1998.

[5] G. J. Foschini, "Layered space-time architecture for wireless communications in a fading environment using multi-element arrays," Bell Labs Technical Journal, vol. 1, pp. 41-59, Autumn 1996.

[6] G. D. Golden, G. J. Foschini, R. A. Valenzuela, and P. W. Wolniansky, "Detection algorithm and initial laboratory results using v-blast spacetime communication architecture," Electronics Letters, vol. 35, pp. 14 16, January 1999.

[7] I. E. Telatar, "Capacity of multi-antenna Gaussian channels," European Transactions on Telecommunications, vol. 10, pp. 585-595, May 1999.

[8] A. S. Dakdouki, V. L. Banket, N. K. Mykhaylov, and A. A. Skopa, "Downlink processing algorithms for multi-antenna wireless communications," IEEE Communications Magazine, vol. 43, pp. 122 - 127, January 2005.

[9] J. S. Blogh and L. Hanzo, Third-generation systems and intelligent wireless networking: smart antennas and adaptive modulation. John Wiley-IEEE Press, 2002.

[10] G. Lebrun, J. Gao, and M. Faulkner, "MIMO transmission over a timevarying channel using SVD," IEEE Transactions on Wireless Communications, vol. 4, pp. 757- 764, March 2005.

[11] T. Dahl, N. Christophersen, and D. Gesbert, "Blind MIMO eigenmode transmission based on the algebraic power method," IEEE Transactions on Signal Processing, vol. 52, pp. 2424- 2431, September 2004.

[12] A. S. Y. Poon, D. N. C. Tse, and R. W. Brodersen, "An adaptive multiantenna transceiver for slowly flat fading channels," IEEE Transactions on Communications, vol. 51, pp. 1820 - 1827, November 2003.

[13] B. Yang, "Projection approximation subspace tracking," IEEE Transactions on Wireless Communications, vol. 43, pp. 95 - 107, January 1995.

[14] X. Wang and H. V. Poor, "Blind multiuser detection: a subspace approach," IEEE Transactions on Information Theory, vol. 44, pp. 677690, March 1998.

[15] C. Li and X. Wang, "Performance comparisons of MIMO techniques with application to WCDMA systems," EURASIP Journal on Applied Signal Processing, vol. 2004, pp. 649-661, May 2004.

[16] J. G. Proakis, Digital Communications. McGraw Hill, 4th ed., 2000. 